\title{
Long-Term Monoacylglycerol Lipase Inhibitor Treatment Decelerates Pathological Changes in APP/PS1-21 Mice, but Behavioral Improvements Require Early-Stage Treatment Onset-Short Report
}

\author{
Rea Pihlaja ${ }^{1,2 *}$, Noora Lindgren ${ }^{3}$, Annamari Torittu ${ }^{4}$, Anniina Snellman1,2, \\ Merja Haaparanta-Solin ${ }^{1,2}$, Juha O. Rinne ${ }^{3,5}$ \\ ${ }^{1}$ PET Preclinical Laboratory, Turku PET Centre, University of Turku, Turku, Finland \\ ${ }^{2}$ Medicity Research Laboratory, University of Turku, Turku, Finland \\ ${ }^{3}$ Turku PET Centre, Turku University Hospital, Turku, Finland \\ ${ }^{4}$ Department of Biochemistry, University of Turku, Turku, Finland \\ ${ }^{5}$ Division of Clinical Neurosciences, Turku University Hospital, Turku, Finland \\ Email: *rea.pihlaja@utu.fi
}

How to cite this paper: Pihlaja, R., Lindgren, N., Torittu, A., Snellman, A., Haaparanta-Solin, M. and Rinne, J.O. (2018) Long-Term Monoacylglycerol Lipase Inhibitor Treatment Decelerates Pathological Changes in APP/PS1-21 Mice, but Behavioral Improvements Require Early-Stage Treatment Onset-Short Report. World Journal of Neuroscience, $\mathbf{8}$, 157-170.

https://doi.org/10.4236/wjns.2018.82014

Received: January 19, 2018

Accepted: May 4, 2018

Published: May 7, 2018

Copyright $\odot 2018$ by authors and Scientific Research Publishing Inc. This work is licensed under the Creative Commons Attribution International License (CC BY 4.0).

http://creativecommons.org/licenses/by/4.0/ (c) (i) Open Access

\begin{abstract}
The arachidonic acid (AA) pathway produces several essential proinflammatory eicosanoids. However, in many neurodegenerative diseases, e.g. Alzheimer's disease $(\mathrm{AD})$, this pathway is chronically hyperactivated. In brain, primarily monoacylglycerol lipase (MAGL) hydrolyzes the endocannabinoid 2-arachidonoylglycerol to AA, which is further metabolized to generate many proinflammatory eicosanoids. MAGL inhibition, simultaneously reducing the level of eicosanoids and increasing those of neuroprotective endocannabinoids, has proved efficacious in some $\mathrm{AD}$ models, reducing neurotoxic $\beta$-amyloid $(\mathrm{A} \beta)$ levels and improving memory functions. Here, a MAGL inhibitor, JZL184 was chronically administered ( $16 \mathrm{mg} / \mathrm{kg}$, i.p., $3 \mathrm{x} /$ wk for $5 \mathrm{mo})$ for $1-1.5 \mathrm{mo}$ and $7-8 \mathrm{mo}$ old transgenic (TG) and wild-type (WT) APP/PS1-21 mice modelling cerebral amyloidosis. According to immunohistochemistry, JZL184 significantly increased the expression levels of cannabinoid receptor 1 in older WT and younger TG and WT mice, decreased cannabinoid receptor 2 and oligomeric $\mathrm{A} \beta$ in older and younger TG mice and decreased microglia-specific marker Ibal in younger TG mice, compared to TG mice treated with vehicle only. However, in the Morris Water Maze test, spatial memory functions improved significantly only in younger TG and WT mice, compared to vehicle-treated littermates. These tentative results suggest
\end{abstract}


that chronic, rather long-term MAGL inhibition can decelerate pathological changes in TG APP/PS1-21 mice but it improves memory functions only when administered at an early stage of the pathology.

\section{Keywords}

Alzheimer's Disease, Arachidonic Acid Pathway, Cannabinoid Receptor, Monoacylglycerol Lipase, Neuroinflammation

\section{Introduction}

Neuroinflammation plays a prominent role in many nervous system pathologies, contributing to the disease process itself, although there probably are differences in certain features in the mediators of inflammation between these diseases [1] [2]. During the progression of Alzheimer's disease (AD), chronically hyperactivated microglia recruit increased numbers of reactive astrocytes and these cells enhance the inflammatory response, leading to the appearance of one of the typical pathological hallmarks of the disease; extracellular $\beta$-amyloid (A $\beta)$ deposits [3]. Reactive astrocytes and microglia begin to proliferate and secrete a wide array of chemokines, inflammatory cytokines and prostaglandins [4] which further stimulate $\mathrm{A} \beta$ production and deposition and induce neuronal dysfunction. There is convincing evidence that the unbalanced inflammatory response occurs relatively early, before $\mathrm{A} \beta$ plaques are deposited, and furthermore, that the suppression of inflammatory signalling exerts beneficial effects [5] [6]. Despite the recent progress regarding $\mathrm{A} \beta$ antibody therapies, these approaches do not yet combat other features of $\mathrm{AD}$ related pathology, e.g. chronic inflammatory responses [7].

The endocannabinoid system is involved in many physiological processes, including cognitive functions, memory and inflammation. This system consists of endogenous signalling lipids i.e. 2-arachidonoylglycerol (2-AG) and $\mathrm{N}$-arachidonoylethanolamine, which bind to and activate cannabinoid receptors $\mathrm{CB} 1$ (CB1R) and CB2 (CB2R) [8]. CB1 expression is abundant in the CNS and its activation controls many normal cellular functions while $\mathrm{CB} 2$ is expressed in immune cells and in the brain in macrophage-derived microglia, mainly during neuroinflammatory responses and neurodegenerative diseases [9] [10]. Activation of $\mathrm{CB} 1$ and $\mathrm{CB} 2$ by $2-\mathrm{AG}$ has been shown to act as a protective neuromodulator against an $\mathrm{A} \beta$ insult [11]. In addition, activation of $\mathrm{CB} 1 / \mathrm{CB}_{2}$ receptors by synthetic agonists has been reported to reduce neuroinflammation and inhibit the production of $\mathrm{A} \beta$ as well as improving cognition in mouse model of $\mathrm{AD}[12]$.

2-AG is the most abundant endocannabinoid molecule; it has equal activating affinity for both $\mathrm{CB} 1$ and $\mathrm{CB} 2$ [13]. The inactivation of 2-AG is almost entirely attributable to the serine hydrolase, monoacylglycerol lipase (MAGL), which accounts for $85 \%$ of 2-AG hydrolysis [14]. MAGL hydrolyses 2-AG to arachi- 
donic acid (AA) which can then be further metabolised to generate many proinflammatory eicosanoids in the brain, liver and the lung [15]. Inhibition of MAGL by genetic manipulation or administration of an MAGL inhibitor reduced the levels of AA and its metabolites prostaglandin E2 and prostaglandin D2 and increased the level of 2-AG and thus the level of neuroprotective endocannabinoids in the brain. Oral administration of an MAGL inhibitor, JZL184, conferred neuroprotection in a parkinsonian mouse model and alleviated LPS-induced neuroinflammation. According to findings emerging from animal models of $\mathrm{AD}$, pharmacological [16] [17] and genetic [18] inactivation of MAGL can reduce the $\mathrm{A} \beta$ load, inhibit neuropathology and improve cognitive functions. It has also been postulated that some of the anti-inflammatory and neuroprotective effects of 2-AG may be through other than CB receptors [16] [18] [19], but the route through which this mechanism acts is still not fully understood and requires clarification.

The present study set-up was an extension to the previous studies from the field, where anti-inflammatory and memory-improving effects of JZL184 were observed in another $\mathrm{AD}$ mouse model. In our study, the anti-inflammatory effects of long-term JZL184-treatment were tentatively tested in transgenic (TG) APP/PS1-21 mice during early- and late-stage proinflammatory phases, and observing the effect on spatial memory functions and the level of activated glial cells, oligomeric $\mathrm{A} \beta$ burden and $\mathrm{CB} 1 \mathrm{R}$ and $\mathrm{CB} 2 \mathrm{R}$ activation by immunohistochemistry.

\section{Materials \& Methods}

\subsection{Animals and Treatments}

The mice were maintained in individual cages under standard conditions $\left(19^{\circ} \mathrm{C}\right.$ $-21^{\circ} \mathrm{C}$, humidity $50 \%-60 \%$, lights on at 7 a.m.-7 p.m., freely available soy-free food (RM3 (E) soya, 801710, Special Diets Service, Essex, UK) and water (RM3 (E) soya, 801710, Special Diets Service, Essex, UK) in the Central Animal Laboratory of the University of Turku. This study was carried out in accordance with the recommendations of the International Council of Laboratory Animal Science (ICLAS). The protocol was approved by the National Animal Experiment Board, Finland (permit number ESAVI/8303/04.10.07/2013). Turku University standard operation procedures \#7001 and \#7601 regarding handling and discarding biohazardous material were followed throughout the study.

TG mice modelling cerebral amyloidosis [C57BL/6J-Tg(Thy- 1-APPSwe, Thy1-PSEN $1{ }^{\star}$ L166P)21]) were used in this study; these animals have a coexpression of human amyloid precursor protein (APP) carrying the K670N/M671L "Swedish" double mutation and human presenilin-1 carrying L166P mutation with a 3-fold overexpression of human APP over endogenous mouse APP. The transgene is expressed under the control of a neuron-specific mThy-1 promoter element, generated on a C57Bl6 background [20].

The JZL184 (Cayman Tocris, USA) formulation was prepared in sa- 
line:emulphor:ethanol (18:1:1 vol/vol/vol) solution as previously described [21]. A dose of $16 \mathrm{mg} / \mathrm{kg}$ JZL184 or vehicle was injected i.p. into 7 - 8 mo old or 1 1.5 mo old TG APP/PS1-21 mice, $3 \mathrm{x} /$ week for 5 mo (Table 1). For the sake of clarity, the groups are designated later as "old" and "young", respectively. Vehicle-treated TG APP/PS1-21 mice or JZL184- or vehicle-treated WT littermates were used as controls.

\subsection{Morris Water Maze}

Animals were allowed to become accustomed to the test room for $1 \mathrm{~h}$ before the actual experiment. EthoVision XT 10 video tracking system (Noldus) was used for tracking and analysing the behavior. The room was dimly lit with indirect white light (app. 50 lux), the test environment was quiet, the visual cues remained unchanged and the observer was not visible to the mouse during the trials.

The Morris Water Maze test, MWM [22] [23] was performed for the mice between 1 p.m-5 p.m. The test included white wading pool, diam. $120 \mathrm{~cm}$ and movable, transparent platform, diam. $13 \mathrm{~cm}$. The platform was submerged $1 \mathrm{~cm}$ under the water level during the test, but removed during the probe trial (last day 6). The mice were allowed $2 \mathrm{~d}$ pretraining ( 2 trials/mouse/d) using separated training alley in the pool, with no visual cues. Pretraining sessions typically reduce stress and habituate the mice to water and searching and climbing onto the platform [23]. After pre training, actual test started using the submerged, hidden platform and providing visual cues for navigation. To avoid hypothermia, which usually impairs movement and learning, the water temperature was kept constantly at $25^{\circ} \mathrm{C}$. Each mouse swam 5 trials per day, starting from different points $(\mathrm{N}, \mathrm{E}, \mathrm{S}, \mathrm{W})$ each time and the order of the starting points was changed every day. The last trial of the last day was the probe trial when there was no platform present. In probe trials, the platform was removed and the animal stayed in the water maze until the maximum trial duration $(1 \mathrm{~min})$ was reached. The spatial learning was examined by observing the path length and escape latency. In the probe trial, time and path length in every quadrant, time in target quadrant and platform crossings were analysed. After the trials, the mice were

Table 1. Animal and treatment data. $\mathrm{TG}=$ transgenic, $\mathrm{WT}=$ wild-type.

\begin{tabular}{lllll}
\hline Genotype & Onset age, mo & Treatment $\mathbf{3} \mathbf{x} /$ wk for $\mathbf{5}$ mo & Sex, $\mathbf{N}$ & N, total \\
\hline TG & $7-8$ & JZL184, 16 mg/kg & $3 \mathrm{M}, 4 \mathrm{~F}$ & 7 \\
TG & $7-8$ & Vehicle & $2 \mathrm{M}, 6 \mathrm{~F}$ & 8 \\
WT & $7-8$ & JZL184, $16 \mathrm{mg} / \mathrm{kg}$ & $8 \mathrm{M}, 3 \mathrm{~F}$ & 11 \\
WT & $7-8$ & Vehicle & $4 \mathrm{M}, 6 \mathrm{~F}$ & 10 \\
TG & $1-1.5$ & JZL184, $16 \mathrm{mg} / \mathrm{kg}$ & $3 \mathrm{M}, 4 \mathrm{~F}$ & 7 \\
TG & $1-1.5$ & Vehicle & $3 \mathrm{M}, 2 \mathrm{~F}$ & 5 \\
WT & $1-1.5$ & JZL184, $16 \mathrm{mg} / \mathrm{kg}$ & $3 \mathrm{M}, 5 \mathrm{~F}$ & 8 \\
WT & $1-1.5$ & Vehicle & $2 \mathrm{M}, 6 \mathrm{~F}$ & 8 \\
\hline
\end{tabular}


terminally anesthetized and transcardially perfused with heparinized saline. The brain was isolated, post-fixed with $4 \%$ paraformaldehyde solution for $24 \mathrm{~h}$, cryoprotected in $30 \%$ sucrose for $48 \mathrm{~h}$, snap frozen in liquid nitrogen and stored at $-70^{\circ} \mathrm{C}$.

\subsection{Immunohistochemistry}

The brain sample was thawed from $-70^{\circ} \mathrm{C}$ to $-20^{\circ} \mathrm{C}$ for $1 \mathrm{~h}$ before cutting the brain sections with a cryostat microtome. Then, $20 \mu \mathrm{m}$ thick coronal sections were cut throughout frontal cortex and hippocampal formation into anti-freeze solution and stored at $-20^{\circ} \mathrm{C}$ until use. Before staining, the sections were washed with $1 \times$ $\mathrm{PB} \mathrm{pH}$ 7.6, shaken gently for $5 \times 30 \mathrm{~min}$ at $\mathrm{RT}$, then $\mathrm{O} / \mathrm{N}$ with slow shaking at $+4^{\circ} \mathrm{C}$ and finally for $3 \times 30 \mathrm{~min}$ at RT. The sections were transferred onto glass slides, air dried for $15 \mathrm{~min}$, rehydrated in $1 \mathrm{x}$ phosphate buffered saline (PBS) for 5 min and permeabilized and blocked using 0.05\% Tween-20 in PBS (PBST; $3 \times 5$ $\min$ ) or $1 \%$ BSA and $2 \%$ NGS with $0.3 \%$ Triton-X in PBS for $1 \mathrm{~h}$, followed by incubation with primary antibody, wash with PBST $(3 \times 5 \mathrm{~min}, \mathrm{RT})$, incubation with secondary antibody, air drying and mounting in Vector HardSet fluorescence mounting medium with DAPI (VectaShied, Vector Laboratories).

To detect the level of activated astrocytes and microglia, the sections were incubated with rabbit anti-GFAP pAb (DAKO, 1:600) and rabbit anti-Iba1 pAb (WAKO, 1:600) in $5 \%$ NGS in PBST overnight at $+4^{\circ} \mathrm{C}$. Furthermore, together with both Ibal and GFAP staining, double staining with mouse $\mathrm{A} \beta$ oligomer-specific mAb, OMAB (Agrisera, 1:700) was performed to determine the level of A $\beta$ oligomers. Secondary antibodies Goat Anti-Rabbit IgG $(\mathrm{H}+\mathrm{L})$ Alexa 568 (Molecular Probes, 1:500) and Donkey Anti-Mouse pAb IgG Dylight 488 (Abcam, 1:800) were incubated on the sections for $2 \mathrm{~h}$, RT. To detect the expression level of cannabinoid receptors, the sections were incubated with rabbit anti-cannabinoid receptor I pAb (Abcam, 1:1000) and rabbit CNR2/CB2 pAb, (Bioss, $1: 1000$ ) in $5 \%$ NGS in PBST overnight at $+4^{\circ} \mathrm{C}$. The secondary antibody, Goat Anti-Rabbit IgG $(\mathrm{H}+\mathrm{L}$ ) Alexa 568 (Molecular Probes, 1:600), was incubated on the sections for $2 \mathrm{~h}, \mathrm{RT}$.

Images were acquired in a Axiovert $200 \mathrm{M}$ fluorescence microscope with a Zeiss AxioCam MRc ccd camera and Zeiss Axiovision software (Zeiss, Germany) or Pannoramic Midi FL Slide Scanner and software Pannoramic Viewer v.1.15.4 (3DHistech Ltd. Hungary), by taking 3 images/animal from 4 different areas. The areas examined were hippocampus (HC), cortex (CTX), striatum (STR) and whole hemisphere (HP). The quantification was performed using per area (\%) method (Image J 1.43 U, Wayne Rasband, NIH, USA). The acquisition of images was performed in a single session, keeping settings constant throughout the quantification procedure. The total number of animals used for immunohistochemical analysis is listed in Table 2.

\subsection{Statistics}

The results are reported as means $\pm \mathrm{SD}$. The data from immunohistochemistry 
Table 2. Animals used in the immunohistochemistry analysis. $\mathrm{TG}=$ transgenic, $\mathrm{WT}=$ wild-type.

\begin{tabular}{lllll}
\hline Genotype & Age, mo & Treatment & Sex, N & N, total \\
\hline TG & $12-13$ & JZL184 & $3 \mathrm{M}, 3 \mathrm{~F}$ & 6 \\
TG & $12-13$ & Vehicle & $2 \mathrm{M}, 5 \mathrm{~F}$ & 7 \\
WT & $12-13$ & JZL184 & $5 \mathrm{M}, 1 \mathrm{~F}$ & 6 \\
WT & $12-13$ & Vehicle & $3 \mathrm{M}, 3 \mathrm{~F}$ & 6 \\
TG & $6-6.5$ & JZL184 & $3 \mathrm{M}, 3 \mathrm{~F}$ & 6 \\
TG & $6-6.5$ & Vehicle & $2 \mathrm{M}, 3 \mathrm{~F}$ & 5 \\
WT & $6-6.5$ & JZL184 & $2 \mathrm{M}, 4 \mathrm{~F}$ & 6 \\
WT & $6-6.5$ & Vehicle & $1 \mathrm{M}, 5 \mathrm{~F}$ & 6 \\
\hline
\end{tabular}

were quantified with two-way ANOVA with Bonferroni post-test using Graph Pad Prism 5, v. 5.01. In MWM analysis, repeated-measures ANOVA were used when comparing differences in latency or path lengths. For data originating from the MWM probe test, one-way ANOVA with Tukey's multiple comparison test was used. In all tests, JZL184-treated TG or WT mice were compared to their vehicle-treated groups. Significance was assumed if $p<0.05$.

\section{Results}

Old (age 7 - $8 \mathrm{mo}$ ) or young (age 1 - $1.5 \mathrm{mo}$ ) TG APP/PS1-21 mice or their WT littermates were treated with JZL184 (16 mg/kg, i.p.) or vehicle, $3 \mathrm{x} /$ week for $5 \mathrm{mo}$.

The MWM was performed when the age of the old and young groups reached app. 12 - 13 mo and $6-6.5$ mo, respectively. Treatment with MAGL inhibitor JZL184 evoked a positive effect on spatial memory functions in young but not in old TG APP/PS1-21 mice. Thus, 5 mo pre-treatment with JZL184 shortened the escape latency (Figure 1(B)) and increased significantly the frequency of target area crossings during probe trials in young TG and WT APP/PS1-21 mice ( $\mathrm{p}<$ 0.01 and $\mathrm{p}<0.05$, respectively) compared to their vehicle-treated littermates (Figure 1(D)). In general, the escape latency for TG mouse groups was longer compared to WT mice throughout the trials, but in young JZL184-treated mice, the escape latency decreased rapidly to the level of WT group performance. In old JZL184-treated TG mice, the escape latency improved but was in general, very long, starting from day 1 in comparison with the other groups (Figure $1(\mathrm{~A})$ ). There was no significant difference in the swimming velocity between groups.

After MWM tests, the mice were sacrificed and the samples for immunohistochemical analysis were collected. According to the results, the level of CB1R in TG mice was generally higher than in WT mice, and in the old mouse group as compared to the young mouse group (Figure 2(A) and Figure 2(D)). The level of CB1R expression increased significantly in old WT, but not in TG mice in all brain areas examined, compared to vehicle-treated (Figure $2(\mathrm{~A}), \mathrm{p}<0.01$ ). 

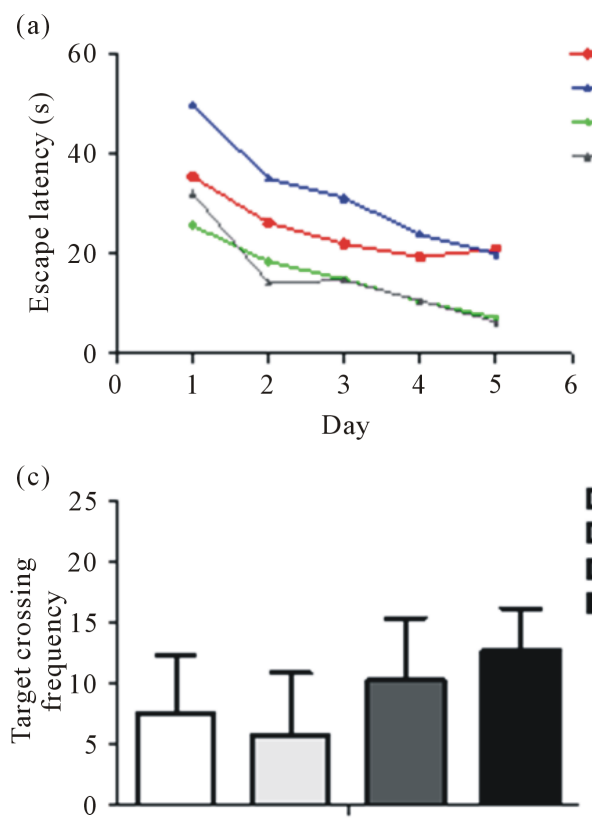

(b)

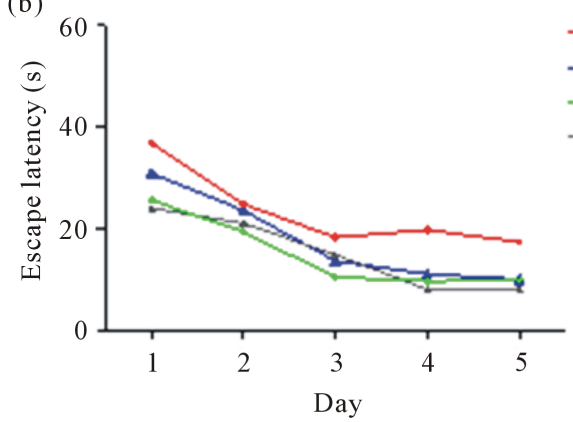

$\rightarrow$ TG veh young -TG JZL 184 young $\rightarrow$ WT veh young -WT JZL184 young

Figure 1. According to the MWM study, pre-treatment with the MAGL inhibitor, JZL184 (16 mg/kg, 3 x/wk for $5 \mathrm{mo}$ ) (B) shortened slightly but non-significantly the escape latency and (D) increased significantly the frequency of target area crossings ( $\mathrm{p}<$ 0.01 and $\mathrm{p}<0.05$, respectively) of $6-6.5$ mo old TG and WT APP/PS1-21 mice, compared to vehicle-treated littermates. However, JZL184 treatment did not have any effect on the performance of $12-13$ mo old TG or WT mice (A), (C). n = $6-8$.

Figure 2(B) and Figure 2(C) represent the level of CB1R immunoreactivity in old vehicle and JZL184 treated TG mice, respectively. In young TG and WT APP/PS1-21 mice, JZL184 increased CB1R expression significantly (Figure 2(D), $\mathrm{p}<0.05)$ compared to vehicle-treated mice. Figure 2(E) and Figure 2(F) represent the level of CB1R immunoreactivity in young vehicle and JZL184 treated TG mice, respectively. Overall, the within-group variation in every CB1R measurement was too large to observe the effect separately in different areas, only in cortex of the young TG mice there was a statistically significant difference (Figure 2(D), $\mathrm{p}<0.05$ ).

JZL184 decreased significantly the level of CB2R expression in the brain of old (Figure 2(G) and Figure 2(I), p < 0.001) and young (Figure 2(J) and Figure $2(\mathrm{~L}), \mathrm{p}<0.05)$ TG mice compared to vehicle-treated mice (Figure $2(\mathrm{G})-(\mathrm{H})$ and Figure $2(\mathrm{~J})-(\mathrm{K}))$. Once again, the within-group variation was too large to observe the effect separately in different brain areas except in the striatum of the old TG mice (Figure 2(G), p < 0.001). The CB2R level was considerably lower in WT mice in both old and young groups compared to TG mice and this situation remained regardless of the treatment.

The level of $\mathrm{A} \beta$ oligomers (OMAB immunoreactivity) decreased significantly in both old (Figure $3(A)$ and Figure $3(C), p<0.001$ ) and young (Figure $3(D)$ and Figure $3(\mathrm{~F}), \mathrm{p}<0.01)$ TG mouse groups in all brain areas examined, when compared to TG mice treated with vehicle alone (Figure 3(A), Figure 3(B), Figure 3(D), Figure 3(E)). However, the within-group variation was too large to observe the effect separately in different areas, with the exception of striatum in 

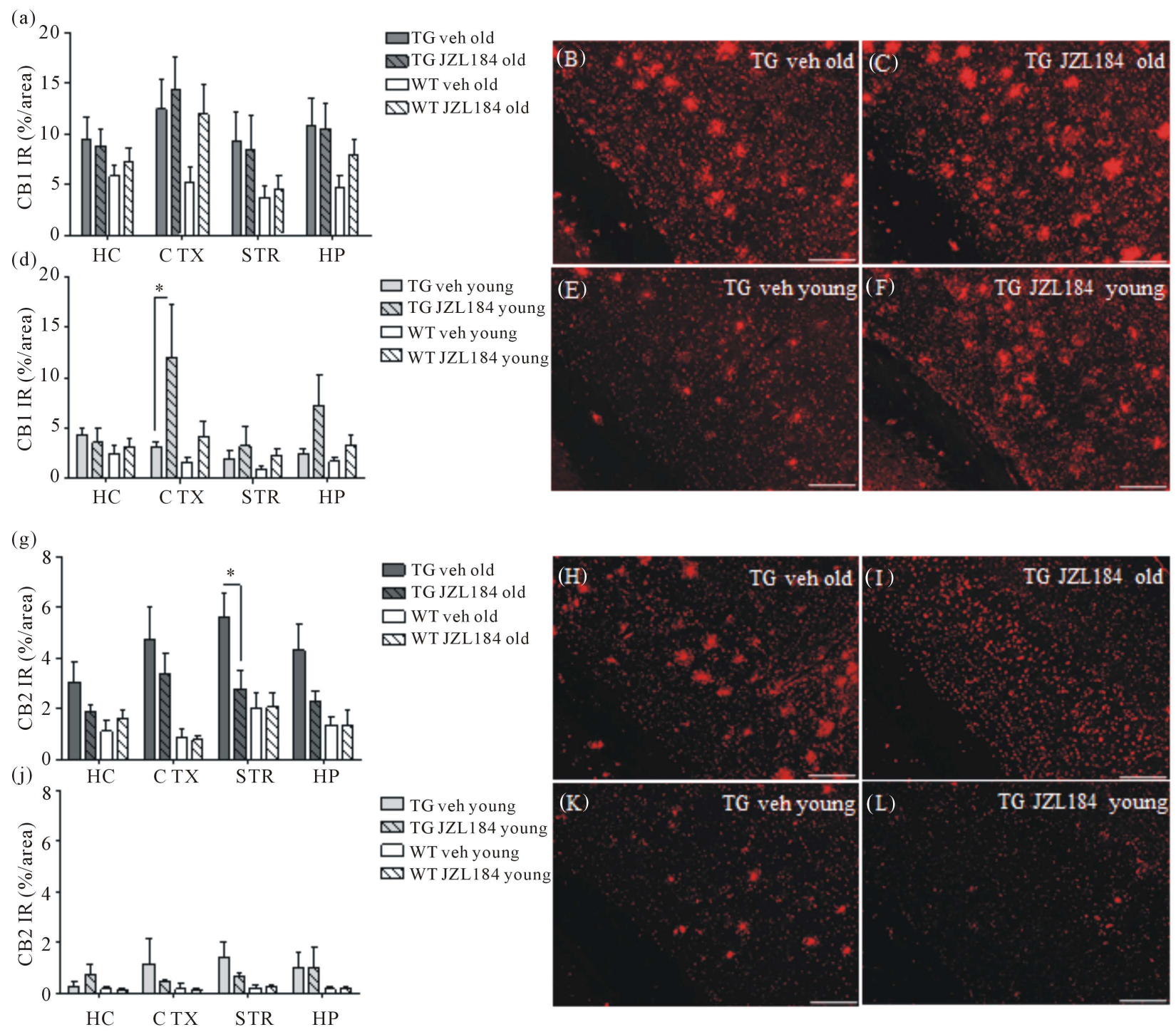

Figure 2. After 5 mo treatment with JZL184, (A), (D) the level of CB1R in TG mice was higher than in WT mice, and in the old group as compared to the young. (A) The level of CB1R expression increased significantly in old WT, but not in TG mice in all brain areas examined, compared to vehicle-treated $(\mathrm{p}<0.01)$. (B), (C) The level of CB1R immunoreactivity in old vehicle and JZL184 treated TG mice. (D) In young TG and WT APP/PS1-21 mice, JZL184 increased CB1R expression significantly compared to vehicle-treated mice ( $\mathrm{p}<0.05)$. (E), (F) The level of CB1R immunoreactivity in young vehicle and JZL184 treated TG mice. JZL184 decreased significantly the level of CB2R expression in all brain areas in old TG mice (G, I, p < 0.001) compared to vehicle-treated mice $(\mathrm{G}),(\mathrm{H})$. In young TG mice, JZL184 treatment decreased the level of CB2R significantly $(\mathrm{J}, \mathrm{L}, \mathrm{p}<0.05)$ compared to vehicle-treated mice $(\mathrm{J}),(\mathrm{K})$. The within-group variation in every measurement was too large to observe the effect separately in different areas, only in (D) cortex of the young TG mice was there a statistically significant difference in CB1R immunoreactivity $(\mathrm{p}<0.05)$ and in $(\mathrm{G})$ striatum of the old TG mice in CB2R immunoreactivity $(\mathrm{p}<0.001) . \mathrm{n}=4-7$. Fluorescence microscopy images represent external capsule and primary somatosensory cortex area. Scale bars indicate $50 \mu \mathrm{m}$. HC = hippocampus, CTX = cortex, STR = striatum, HP = hemisphere.

old TG mouse group (Figure 3(A), $\mathrm{p}<0.01$ ). The level of the activated microglia (Iba1 immunoreactivity) decreased only slightly in old TG mice (Figure 4(A) and Figure 4(C)) but significantly in young TG mice (Figure 4(D) and Figure $4(\mathrm{~F}), \mathrm{p}<0.01)$, when compared to TG mice treated with vehicle alone 

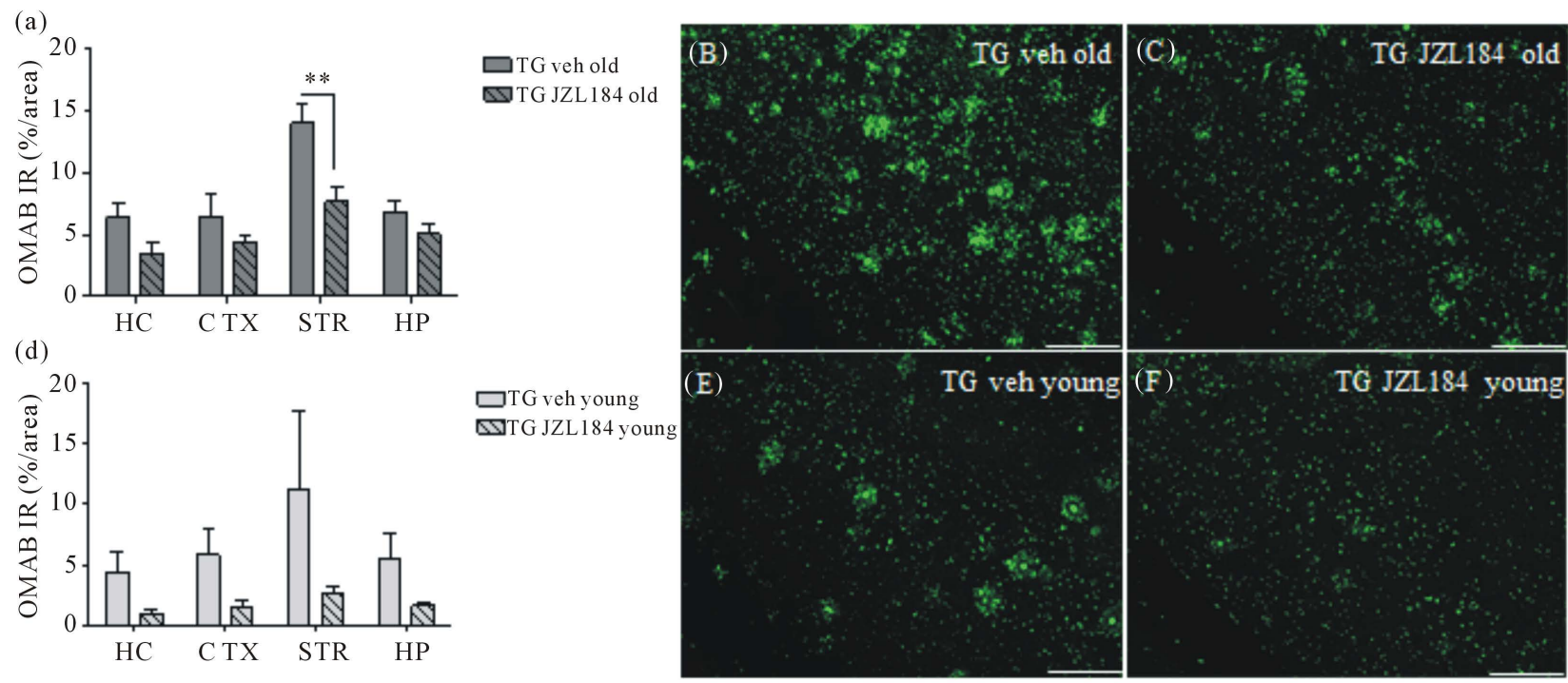

Figure 3. After 5 mo treatment with JZL184, the level of A $\beta$ oligomers (OMAB immunoreactivity) decreased significantly in (A), (C) old ( $<<0.001)$ and (D), (F) young ( $<0.01)$ TG mouse groups in all brain areas examined, when compared to age-matched TG mice treated with vehicle only (A), (B), (D), (E). $n=4-6$. The within-group variation was too large to allow an assessment of the effect separately in the different brain areas, with the exception of the striatum in old TG mouse group (A), p $<0.01)$. Fluorescence microscopy images represent external capsule and primary somatosensory cortex area. Scale bars indicate $50 \mu \mathrm{m}$. $\mathrm{HC}=$ hippocampus, CTX = cortex, STR = striatum, HP = hemisphere.

(Figure 4(A), Figure 4(B), Figure 4(D), Figure 4(E)). Again, the within-group variation was too large to observe the effect separately in different areas, with the exception of the cortex (Figure 4(D), $\mathrm{p}<0.01$ ). In general, the level of astrocyte specific GFAP was higher in TG mice when compared to WT mice (Figure 4(G) and Figure $4(\mathrm{~J})$ ), but the level of expression did not change in any of the groups due to JZL184 treatment (Figure 4(G)-(L)).

\section{Discussion}

Consistent with previous reports in $\mathrm{AD}$ animal models concerning long-term JZL184 treatment [16] or genetic inactivation of MAGL [18], our results showed that JZL184 reduced proinflammatory responses and improved spatial memory.

According to the immunohistochemical analysis, the expression of both CB1R and CB2R was higher in TG compared to WT mice. MAGL inhibition induced a notable increase in CB1R expression in young TG mice and reduced significantly the level of CB2R expression both in young and old TG mice, when compared to their vehicle-treated littermates. CB2R are typically inflammation-induced, mainly microglia-specific, receptors [24]. Indeed, in our study, MAGL inhibition reduced the microglia-specific, inflammation-related Iba1 both in old, but especially in young TG APP/PS1-21 mice, when compared to vehicle-treated littermates. Furthermore, the levels of $\mathrm{A} \beta$ oligomeric species were simultaneously reduced due to long-term MAGL inhibition. However, despite that the effect of the JZL184 treatment according to histological analysis was significant overall when compared to treatment with vehicle only, the within-group variation in many 

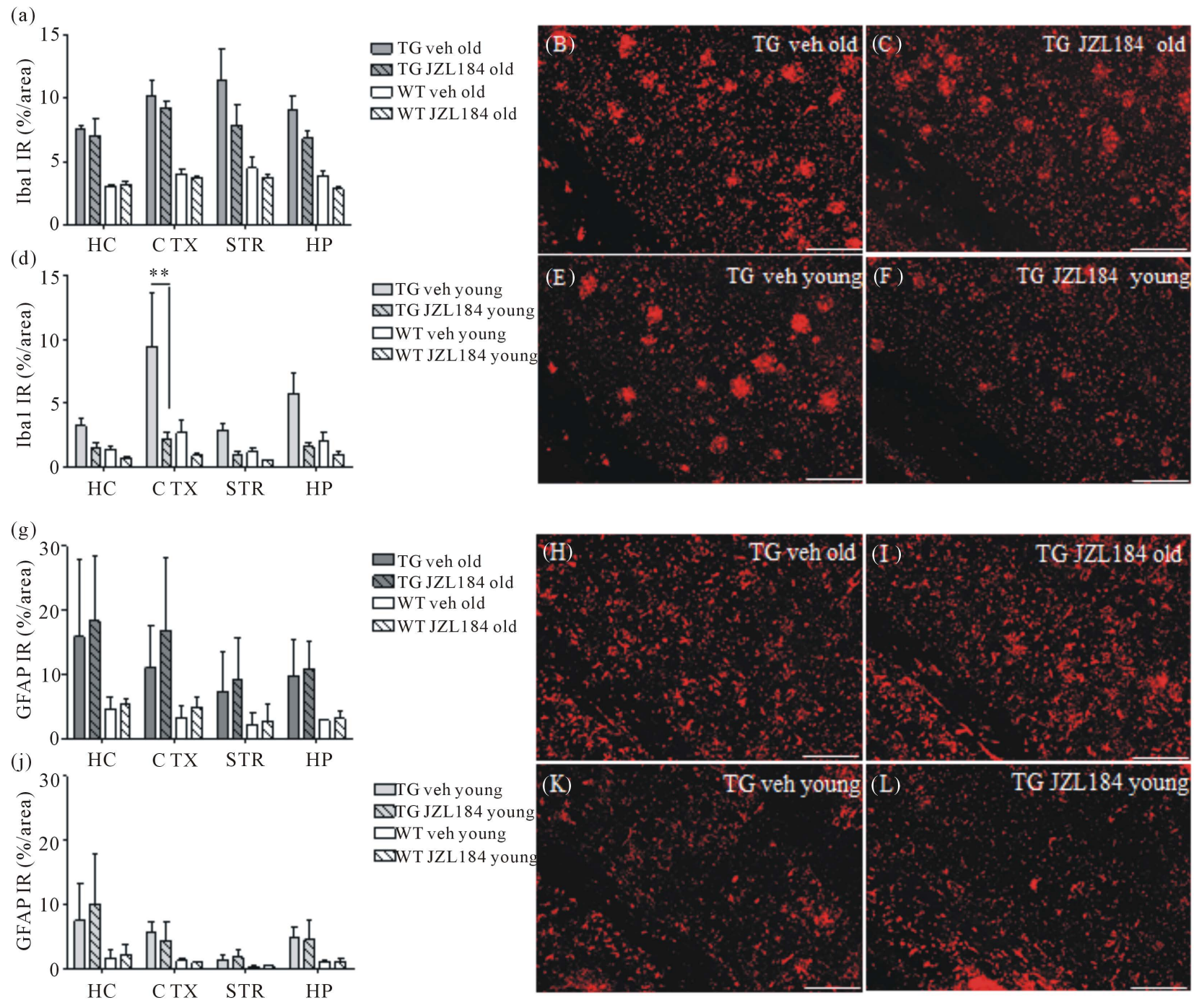

Figure 4. After 5 mo treatment with JZL184, the level of Iba1 decreased (A), (C) slightly but not significantly in old and (D), (F) significantly $(\mathrm{p}<0.01)$ in the young TG APP/PS1-21 mouse group in all brain areas, as compared to TG mice treated with vehicle only (A), (B), (D), (E). The within-group variation was too large to allow an assessment of the effect separately in the different brain areas, with the exception of the cortex in young TG mouse group ((D), p < 0.01). JZL184 treatment did not affect the level of GFAP expression in any of the groups $(\mathrm{G})-(\mathrm{L}) . \mathrm{n}=4-7$. Fluorescence microscopy images represent external capsule and primary somatosensory cortex area. Scale bars indicate $50 \mu \mathrm{m}$. HC = hippocampus, CTX = cortex, STR $=$ striatum, HP = hemisphere.

analysis was too large to observe significant effects separately in different brain areas. Also, we did not have the possibility to separately follow the possible sex-related effects in this study set-up.

We noted that long-term MAGL inhibition attenuated behavioral impairments efficiently when administered during the early stage of the development of $\mathrm{AD}$ pathology. However, it did not improve the performance of the old TG or WT mouse group in MWM, thus showing neither disease-modifying nor pro-cognitive effect. In our study, the treatment with JZL184 in the old mouse group started at the age of $7-8$ months. One straightforward explanation would be that with such a late time point for treatment onset in this mouse model, the 
massive $\mathrm{A} \beta$ accumulation and resulting vicious cycles of proinflammatory reactions in TG mice have already evoked such severe changes that it is impossible to restore the function of the damaged nerve cells, even though the level of microgliosis and the oligomeric forms of $\mathrm{A} \beta$ were reduced according to the results obtained from immunohistochemical analysis. In young APP/PS1-21 mice, JZL184 improved spatial memory functions significantly in TG, but also in WT mice, compared to vehicle treated mice. This finding might suggest only pro-cognitive rather than disease-modifying role of JZL184. But if this would be the situation, also the performance in old WT mice should have been expected to improve significantly. Alternatively, the pro-cognitive effect of MAGL inhibition might be age-dependent. MAGL has already been suggested as one of the most important regulatory enzymes in the inflammatory pathways of the brain, and its inhibition has been shown beneficial, anti-inflammatory effects in certain animal models. Our long-term study set-up performed with an animal model of cerebral amyloidosis supports these previously published results, but as an extension to the previous findings suggests that the sooner the proinflammatory reactions mediated by the glial cells are halted, the more efficient is the capacity of these cells to reduce the levels of the toxic forms of $\mathrm{A} \beta$ and to restore homeostasis of CNS inflammatory systems. However, the results obtained are tentative, requiring larger study set-up with additional methodological backup.

\section{Conflict of Interest}

All authors declare that they have no conflict of interest.

\section{Acknowledgements}

We are grateful to the Medicity Research Laboratory for assistance in research.

\section{Funding}

This work was funded by the Jane and Aatos Erkko Foundation of Finland, State funding for university level health research, Turku University Hospital, Finland and European Community's Seventh Framework Programme (FP7/2007-2013) under grant agreement no. HEALTH-F2-2011-278850 (INMiND).

\section{Author Contributions}

RP designed, performed and analysed the experiments and wrote the manuscript. NL assisted with the MWM experimental setup. AS and AMT assisted with the laboratory experiments. MHS and JR analysed the data and coordinated collaborations. All authors checked and approved the final manuscript.

\section{References}

[1] Schwartz, M. and Deczkowska, A. (2016) Neurological Disease as a Failure of Brain-Immune Crosstalk: The Multiple Faces of Neuroinflammation. Trends Immunol, 37, 668-679. https://doi.org/10.1016/j.it.2016.08.001 
[2] Ransohoff, R.M. (2016) How Neuroinflammation Contributes to Neurodegeneration. Science, 353, 777-783. https://doi.org/10.1126/science.aag2590

[3] Sastre, M., Walter, J. and Gentleman, S.M. (2008) Interactions between APP Secretases and Inflammatory Mediators. Journal of Neuroinflammation, 5, 25. https://doi.org/10.1186/1742-2094-5-25

[4] Heneka, M.T. and O’Banion, M.K. (2007) Inflammatory Processes in Alzheimer's disease. Journal of Neuroinflammation, 184, 69-91. https://doi.org/10.1016/j.jneuroim.2006.11.017

[5] Heneka, M.T., Carson, M.J., El Khoury, J., Landreth, G.E., Brosseron, F., Feinstein, D.L., Jacobs, A.H., Wyss-Coray, T., Vitorica, J., Ransohoff, R.M., Herrup, K., Frautschy, S.A., Finsen, B., Brown, G.C., Verkhratsky, A., Yamanaka, K., Koistinaho, J., Latz, E., Halle, A., Petzold, G.C., Town, T., Morgan, D., Shinohara, M.L., Perry, V.H., Holmes, C., Bazan, N.G., Brooks, D.J., Hunot, S., Joseph, B., Deigendesch, N., Garaschuk, O., Boddeke, E., Dinarello, C.A., Breitner, J.C., Cole, G.M., Golenbock, D.T. and Kummer, M.P. (2015) Neuroinflammation in Alzheimer's Disease. The Lancet Neurology, 14, 388-405. https://doi.org/10.1016/S1474-4422(15)70016-5

[6] Wyss-Coray, T. and Rogers, J. (2012) Inflammation in Alzheimer Disease-A Brief Review of the Basic Science and Clinical Literature. Cold Spring Harbor Perspectives in Medicine, 2, a006346. https://doi.org/10.1101/cshperspect.a006346

[7] Selkoe, D.J. and Hardy, J. (2016) The Amyloid Hypothesis of Alzheimer's Disease at 25 Years. EMBO Molecular Medicine, 8, 595-608. https://doi.org/10.15252/emmm.201606210

[8] Di Marzo, V. and Petrosino S. (2007) Endocannabinoids and the Regulation of Their Levels in Health and Disease. Current Opinion in Lipidology, 18, 129-140. https://doi.org/10.1097/MOL.0b013e32803dbdec

[9] Ashton, J.C. and Glass, M. (2007) The Cannabinoid CB2 Receptor as a Target for Inflammation-Dependent Neurodegeneration. Current Neuropharmacology, 5, 73-80. https://doi.org/10.2174/157015907780866884

[10] Miller, A.M. and Stella, N. (2008) CB2 Receptor-Mediated Migration of Immune Cells: It Can Go Either Way. British Journal of Pharmacology, 153, 299-308. https://doi.org/10.1038/sj.bjp.0707523

[11] Chen, X., Zhang, J. and Chen, C. (2011) Endocannabinoid 2-Arachidonoylglycerol Protects Neurons against $\beta$-Amyloid Insults. Neuroscience, 178, 159-168. https://doi.org/10.1016/j.neuroscience.2011.01.024

[12] Martín-Moreno, A.M., Reigada, D., Ramírez, B.G., Mechoulam, R., Innamorato, N., Cuadrado, A. and de Ceballos, M.L. (2011) Cannabidiol and Other Cannabinoids Reduce Microglial Activation in Vitro and in Vivo: Relevance to Alzheimer's Disease. Molecular Pharmacology, 79, 964-973.

https://doi.org/10.1124/mol.111.071290

[13] Mechoulam, R., Ben-Shabat, S., Hanus, L., Ligumsky, M., Kaminski, N.E., Schatz, A.R., Gopher, A., Almog, S., Martin, B.R., Compton, D.R., et al. (1995) Identification of an Endogenous 2-Monoglyceride, Present in Canine Gut, That Binds to Cannabinoid Receptors. Biochemical Pharmacology, 50, 83-90. https://doi.org/10.1016/0006-2952(95)00109-D

[14] Blankman, J.L., Simon, G.M. and Cravatt, B.F. (2007) A Comprehensive Profile of Brain Enzymes that Hydrolyze the Endocannabinoid 2-Arachidonoylglycerol. Chemistry \& Biology, 14, 1347-1356. https://doi.org/10.1016/j.chembiol.2007.11.006

[15] Nomura, D.K., Morrison, B.E., Blankman, J.L., Long, J.Z., Kinsey, S.G., Marcondes, 
M.C., Ward, A.M., Hahn, Y.K., Lichtman, A.H., Conti, B. and Cravatt, B.F. (2011) Endocannabinoid Hydrolysis Generates Brain Prostaglandins That Promote Neuroinflammation. Science, 334, 809-813. https://doi.org/10.1126/science.1209200

[16] Chen, R., Zhang, J., Wu, Y., Wang, D., Feng, G., Tang, Y.P., Teng, Z. and Chen C. (2012) Monoacylglycerol Lipase Is a Therapeutic Target for Alzheimer's Disease. Cell Reports, 2, 1329-1339. https://doi.org/10.1016/j.celrep.2012.09.030

[17] Pihlaja, R., Takkinen, J., Eskola, O., Vasara, J., López-Picón, F.R., Haaparanta-Solin, M. and Rinne J.O. (2015) Monoacylglycerol Lipase Inhibitor JZL184 Reduces Neuroinflammatory Response in APdE9 Mice and in Adult Mouse Glial Cells. Journal of Neuroinflammation, 12, 81. https://doi.org/10.1186/s12974-015-0305-9

[18] Piro, J.R., Benjamin, D.I., Duerr, J.M., Pi, Y., Gonzales, C., Wood, K.M., Schwartz, J.W., Nomura, D.K. and Samad, T.A. (2012) A Dysregulated Endocannabinoid-Eicosanoid Network Supports Pathogenesis in a Mouse Model of Alzheimer's Disease. Cell Reports, 1, 617-623.

https://doi.org/10.1016/j.celrep.2012.05.001

[19] Grabner, G.F., Eichmann, T.O., Wagner, B., Gao, Y., Farzi, A., Taschler, U., Radner, F.P., Schweiger, M., Lass, A., Holzer, P., Zinser, E., Tschöp, M.H., Yi, C.X. and Zimmermann, R. (2016) Deletion of Monoglyceride Lipase in Astrocytes Attenuates Lipopolysaccharide-Induced Neuroinflammation. Journal of Biological Chemistry, 291, 913-923. https://doi.org/10.1074/jbc.M115.683615

[20] Radde, R., Bolmont, T., Kaeser, S.A., Coomaraswamy, J., Lindau, D., Stoltze, L., Calhoun, M.E., Jäggi, F., Wolburg, H., Gengler, S., Haass, C., Ghetti, B., Czech, C.,

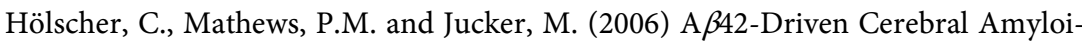
dosis in Transgenic Mice Reveals Early and Robust Pathology. EMBO Reports, 7, 940-946. https://doi.org/10.1038/sj.embor.7400784

[21] Long, J.Z., Nomura, D.K. and Cravatt, B.F. (2009) Characterization of Monoacylglycerol Lipase Inhibition Reveals Differences in Central and Peripheral Endocannabinoid Metabolism. Chemistry \& Biology, 16, 744-753.

https://doi.org/10.1016/j.chembiol.2009.05.009

[22] Morris, R. (1984) Developments of a Water-Maze Procedure for Studying Spatial Learning in the Rat. Journal of Neuroscience Methods, 11, 47-60. https://doi.org/10.1016/0165-0270(84)90007-4

[23] Tanila, H. (2012) Wading Pools, Fading Memorie-Place Navigation in Transgenic Mouse Models of Alzheimer's Disease. Frontiers in Aging Neuroscience, 4, 11. https://doi.org/10.3389/fnagi.2012.00011

[24] Aso, E. and Ferrer, I. (2016) CB2 Cannabinoid Receptor as Potential Target against Alzheimer's Disease. Frontiers in Neuroscience, 10, 243. https://doi.org/10.3389/fnins.2016.00243 


\section{Abbreviations}

2-AG: 2-arachidonoylglycerol

AA: arachidonic acid

APP: amyloid precursor protein

A $\beta$ : beta-amyloid

BSA: bovine serum albumin

CBR: cannabinoid receptor

GFAP: glial fibrillary acidic protein

Iba1: ionized calcium-binding adapter molecule 1

LPS: lipopolysaccharide

mAb: monoclonal antibody

MAGL: monoacylglycerol lipase

MWM: Morris Water Maze

NGS: normal goat serum;

NO: nitric oxide;

OMAB: oligomer-specific monoclonal antibody

pAb: polyclonal antibody 